\title{
Dynamics of students' ontological reasoning across contexts in modern physics
}

\author{
Jessica R. Hoehn, Julian D. Gifford, and Noah D. Finkelstein \\ Department of Physics, University of Colorado, 390 UCB, Boulder, CO 80309
}

\begin{abstract}
The ways in which students organize knowledge about entities, such as photons and electrons, (or their ontologies) influence their learning of quantum phenomena. In prior work, we have described different types of dynamic ontologies seen in collective student reasoning; here we extend this analysis to individual written responses to Modern Physics homework, exam, and survey questions about canonical quantum mechanics topics. We present a refined framework that describes unitary, parallel, and blended ontological structures, and then examine students' patterns of use of ontological structures across entities (e.g., photon or electron) and topic areas (double slit, Mach-Zehnder interferometer, and tunneling). Analysis of seven students' responses demonstrate their flexible use of ontologies in individual written work. Through this analysis, we raise questions for future studies about how the wording and framing of the prompts impacts students' ontological reasoning.
\end{abstract}

\section{INTRODUCTION}

The ways in which students reason about what kind of entity something is (or the ontologies they use) influences the way they learn physics $[1,2]$. Learning QM requires learning how to think about entities in a new way (e.g., an electron, which is classically a particle, now exhibits wavelike properties). We continue prior work [3] that not only contributes to the historical debate about the nature of ontologies [1, 4], but documents the ways in which ontologies can be dynamic. The term "dynamic ontologies" encompasses the ability of both students and experts to move flexibly between different ontologies depending on the context [4], coordinate multiple ontologies to form a new blended ontology [5], and negotiate meaning in the moment [3].

In prior work [3], we conducted analyses of students' collective conversations around ontologies of photons and electrons. Through the development of a framework to delineate two different types of dynamic ontologies - parallel (switching back and forth between ontologies) and blended (creation of a new entity from the combination of multiple input ontologies)—we provided evidence that students are capable of engaging in different forms of dynamic ontological reasoning. We now seek to triangulate these results with additional data sources, to both confirm the applicability of the framework as well as to investigate another side of students' ontological reasoning in QM. This paper addresses three questions: 1) How do we need to modify the initial framework in order to apply it to student reasoning in individual written work? 2) Do we see the same kind of dynamic ontologies in individual written student work that we do in collective oral reasoning? 3) If so, what are the patterns of use of ontologies in these additional contexts? Investigation of these questions leads to a fourth emergent question to be explored in upcoming work: 4) How does the framing of prompts impact students' ontological reasoning? In answering the first two questions, we seek to extend the framework from collective to individual reasoning and from students talking to writing. To do so, we coded multiple-choice and written responses from three additional data sources-homework, survey, and exam questions-across three topic areas in modern physics for the seven participants from the original focus group study.

The purpose of this paper is fourfold. First, we present a refinement of our framework [3] to describe the dynamics of ontologies. Second, we demonstrate that we are able to apply the framework to individual written responses and identify variations in students' ontological structures. Third, we present the results of an initial triangulation analysis across topic areas and data sources. Here, we identify a variety of ontological structures for both photons and electrons within each topic area, and note that blended and parallel ontologies occurred more frequently for photons than electrons. Fourth, we raise questions for further investigation about the impact of the wording and framing of prompts.

\section{BACKGROUND}

In prior work [3], we developed a framework to describe the different kinds of dynamic ontologies, grounded in examples from focus group video data. The goal of this focus group study was to look for and provide evidence of the dynamic nature of students' ontological reasoning, and refine what we meant when saying that ontologies were dynamic. Our focus group participants were students enrolled in a Modern Physics for Engineers course; we had seven students split into two groups, and each group met six times over the course of a semester. During the focus group sessions, students worked on both structured and open-ended problems relating to the course content. They were encouraged to talk to one another and think out loud as they worked, and every session was video recorded. From the video data, we analyzed select episodes using a collective conceptual blending framework [6] in order to map out the ontologies the groups were using as they made sense of quantum phenomena. The three focal episodes of the analysis were canonical topic areas of QM: double slit experiment with a single photon, MachZehnder interferometer with a single photon, and quantum tunneling. From the collective conversations around photons and electrons in these three topic areas, we identified some of the types of ontological reasoning students are capable of (but made no claims about their ubiquity or frequency). The 
initial framework identifies three different types of ontological structures that students may use within a given reasoning episode: blended, parallel, and fixed. A blended ontology combines elements from prior ontologies to create a new ontological category that is a conceptual blend [7]. For particular examples of the conceptual blends, see Ref [3]. In the focus groups, blended ontologies are characterized by in the moment negotiation of meaning. Parallel ontologies involve switching back and forth between two (or more) stable ontologies, but never combining them into a single and new category. The third ontological structure was originally labeled as a "fixed ontology" but in our refinement of the framework resulting from the triangulation study described in this paper we have renamed it "unitary ontology". This represents a single stable ontology applied in a given reasoning episode, but does not exclude flexibility and movement between other ontologies outside of the local reasoning episode.

\section{METHODS}

This paper is one part of a larger study to understand students' ontological reasoning in QM. Here, we refine the prior framework so as to expand the domain of application to include individual written work in addition to collective oral reasoning. In particular, we show that this refined framework is still applicable when we move from collective oral reasoning to individual written responses. In this way, we seek convergence of findings from two different sets of data and analyses. In triangulating the focus group data with additional data sources, we also seek to increase the scope of our understanding of students' ontological reasoning in QM.

This research takes place in a modern physics course primarily for engineering majors that has been transformed over a period of several years. The course focuses on conceptual foundations and real world applications of QM [8] and attends to issues of interpretation of QM [9]. The data in this paper come from one semester of this course when we collected all course artifacts including homework, survey, and exam responses. This is the same semester in which we ran the focus group study with seven students, discussed above. From the larger collection of course artifacts, we identified homework, survey, and exam questions that corresponded to the three topic areas from the focus group episodes (double slit experiment, Mach-Zehnder interferometer, quantum tunneling) and that allowed students to externalize their ontological reasoning. Although we have responses from all 130 students in the course, here we focus our analysis only on responses from the same seven focus group students of the prior study. The participants included six males and one female. Of the six people who provided information about their race and ethnicity, three self-reported as white, one as Hispanic and Caucasian, one as Asian, and one as Japanese, Irish, and German. There are three freshmen, two sophomores, and two juniors.

Based on the original framework, we developed a code- book to code students' responses for the entity (light, photon, or electron) they are reasoning about, the ontology(ies) (wave, particle, both particle/wave, something else, not particle, or not wave) they use to do so, and the ontological structure (unitary, parallel, or blended). See prior work for examples of unitary, parallel, and blended ontologies [3]. The three authors independently coded a small subset of the responses and then discussed each response in detail, iteratively refining both the codebook and the framework itself until we had $100 \%$ agreement. The lead author completed the remainder of the coding, discussing with the other two authors to come to consensus around responses that were difficult to code. When there are two or more questions that, when considered together, give us additional information about the student's ontology or ontological structure, we group them and only count the code for the cluster as a whole. After grouping the questions into clusters, we have 18 distinct items to code and count across the seven students ( 6 for double slit, 2 for tunneling, and 10 for Mach-Zehnder), although not every student answered every question. We then counted the codes for ontology and ontological structure and looked at the results by student, topic area, and entity. When we coded the student responses, we included a "can't tell" code in both ontology and ontological structure, indicating that we did not have enough evidence to code as a specific ontology structure. Only $8.4 \%$ of all responses were coded as "can't tell". We exclude the items that we coded as "can't tell" from the analysis, leaving us with 110 total coded responses. In addition to coding the student responses, we coded the questions themselves for whether we felt that they were leading towards a certain ontology or ontological structure, and if so, what ontology we might expect students to use when answering the question. For example, "How is a photon like a particle?" is leading towards a unitary particle ontology, while "In what ways does a photon behave like a particle and wave at the same time?" is leading away from a unitary ontology.

\section{RESULTS}

The first major outcome of this study was the refinement of our framework for dynamic ontologies. Through the process of developing the codebook, we refined the wording and presentation of the framework itself. In the context of collective reasoning in focus groups, we described blended ontologies as being constructed in the moment, as we could see the negotiation of meaning unfolding (in a messy, and often productive, way) bit by bit in the video. However, when looking at individual written reasoning turned in on a homework, survey, or exam, we cannot comment on in the moment reasoning. In these instances, we do still identify and describe blended ontologies as a new category created by combining elements from multiple pre-existing ontologies. The blended ontologies are still dynamic, though in a different way than when we identify collectively constructed blends unfolding and being negotiated throughout a conversation. This difference comes 


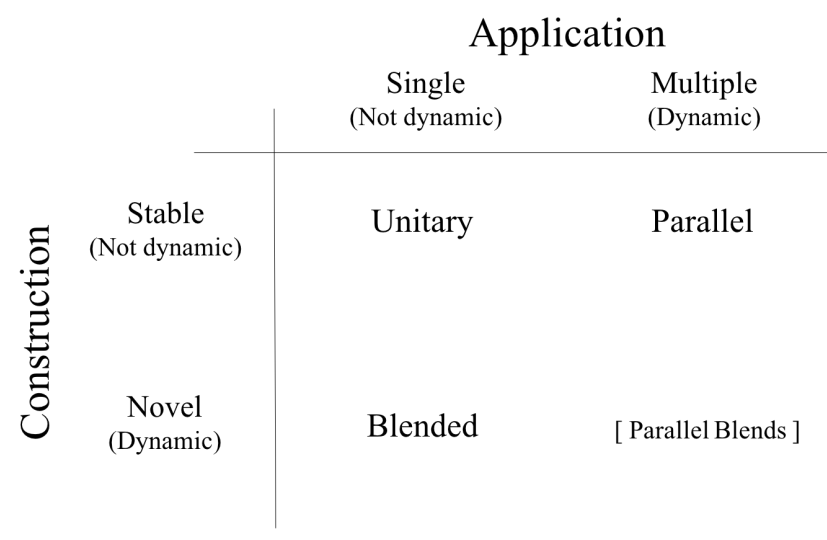

FIG. 1. Refined framework for describing the different types of ontological reasoning. The bottom right entry is in brackets because we do not see this structure in our data.

from the difference between the theory of conceptual blending [7] as it was originally intended to describe the cognition of individuals and the adapted collective conceptual blending framework [6] which treats the blends as collectively constructed and socially distributed. Although the substance of our dynamic ontologies framework has not changed, we no longer label blended ontologies as being constructed in the moment. The refined version of the framework is shown in Figure 1. It describes two aspects of ontologies that may or may not be dynamic: construction and application. Single application of a stable, pre-existing (and perhaps canonical) ontology is a unitary structure. Application of multiple stable ontologies while keeping them separate from one another is a parallel ontology. A single application of a new ontology that can be described as a conceptual blend of other ontologies is a blended ontology. We can imagine the bottom right square of the diagram to be "parallel blends"- switching back and forth between two (or more) new blended ontologies. Due to the timescale of our focus group episodes and small grain size of the responses to homework, survey, and exam questions, we do not see this complex and nuanced ontological structure in our data. However, we can imagine zooming out on a bigger picture of a group's conversation or looking at student responses over time to possibly see such complexity.

We are indeed able to apply this modified framework, initially developed in the context of collective focus group reasoning, to other course artifacts and data sources. We take the low percentage of responses coded as "can't tell" to be a testament to the applicability of our codebook.

The second outcome of this work is that we do see evidence of students' flexible use of ontologies in the individual written responses. Within each entity (photon or electron), students used a variety of ontologies (see Table I). The most common ontology for photons was "both particle and wave", and for electrons it was "wave". All of the wave ontology codes for electrons came from multiple-choice dou- ble slit questions ${ }^{1}$. Overall (aggregating all students for all entities and all topic areas), 56\% of the responses were unitary, $16 \%$ parallel, and $29 \%$ blended. When excluding the multiple-choice questions (5 out of the 18 total questions, 4 for double slit and 1 Mach-Zehnder), which give us less information about a student's actual reasoning, the majority of responses are coded as blended. These results are enumerated in Table II. When separating multiple-choice from written questions, we see that the distributions of ontological structures are statistically significantly different at the $\alpha=0.05$ level. The difference between including and excluding multiple-choice questions in the analysis is due to the fact that multiple-choice questions only allow us to say that a response is consistent with a single ontology and thus all of the multiple-choice questions are coded as unitary ${ }^{2}$. This result foreshadows our emergent fourth research question and the investigation of the impact of framing of the prompts.

TABLE I. Percentages of ontology codes for photons and electrons. $N$ gives the number of responses coded for each entity.

\begin{tabular}{lll}
\hline & Photons $(N=77)$ & Electrons $(N=20)$ \\
\hline Particle & $9 \%$ & $15 \%$ \\
Wave & $26 \%$ & $60 \%$ \\
Both & $43 \%$ & $5 \%$ \\
Something else & $13 \%$ & $20 \%$ \\
Not particle & $0 \%$ & $0 \%$ \\
Not wave & $9 \%$ & $0 \%$ \\
\hline
\end{tabular}

TABLE II. Percentages of ontological structure codes. The distributions for written and multiple-choice questions are statistically significantly different from one another $(p<0.0001)$, as are the distributions for photons and electrons $(p=0.046) . N$ gives the number of responses coded in each category.

\begin{tabular}{lllll}
\hline & $N$ & Unitary & Parallel & Blended \\
\hline Written & 77 & $36 \%$ & $22 \%$ & $42 \%$ \\
Multi. Chc. & 33 & $100 \%$ & $0 \%$ & $0 \%$ \\
All & 110 & $56 \%$ & $16 \%$ & $29 \%$ \\
\hline Photons & 77 & $43 \%$ & $22 \%$ & $35 \%$ \\
Electrons & 20 & $75 \%$ & $0 \%$ & $25 \%$ \\
\hline
\end{tabular}

Addressing the third research question, we looked for patterns of use of the ontological structures. Both blended and parallel ontologies occurred more frequently for photons than for electrons (across all question formats), as shown in Table II. The distributions of ontological structures for photons and electrons are significantly different at the $\alpha=0.05$ level.

\footnotetext{
1 The questions ask what pattern you will see on the screen when sending electrons through the double slit experiment. A correct answer of an interference pattern is consistent with a wave ontology for the electron.

${ }^{2}$ We could, however, imagine writing multiple choice questions for which the answers would be consistent with parallel or blended ontologies.
} 


\section{DISCUSSION \& CONCLUSIONS}

The refinement of the framework allows us to expand the domain of application beyond collective oral reasoning in which it was originally developed. This is a non-trivial finding as the transitions from talking to writing, and from collective to individual both presented potential challenges to the ubiquity of the framework. This framework will be a productive tool to leverage in further studies of students' ontological reasoning. The second outcome is that we do see similar kinds of dynamic ontologies in the individual written work as were present in the prior collective focus group conversations, with seven students using parallel ontologies and five using blended. The presence of multiple ontology codes for both photons and electrons, as well as all three ontological structure codes (unitary, parallel, and blended) suggest that in these contexts students are moving between ontologies in a flexible way.

Addressing the third research question, we identified blended and parallel ontologies more often for photons than for electrons. We are interested to explore the pattern further and see if it persists when responses from the entire class of 130 students are analyzed. The fourth and final outcome is that this work has raised new questions about the impact of the wording and framing of the prompts on students' use of ontologies. Our initial question about the difference in ontological structures for photons and electrons was whether it was due, at least in part, to the types of questions we asked students. We might imagine that students used blended and parallel ontologies more often for photons than electrons because we asked more questions about photons that encouraged them to do so (or asked more questions about electrons that encouraged them to use unitary ontologies). Or, we might imagine that students bring in prior (classical) knowledge of electrons but not for photons and thus they are more likely to be creating new ontologies or moving between multiple classical ontologies in order to make sense of the photon. Preliminary analysis suggests that the difference in frequency of blended and parallel ontologies between photons and electrons is not due to the types of questions. If anything, the difference in framing of the questions goes in the opposite direction. Of the photon questions, $58 \%$ (7 out of 12) were coded as leading towards a specific ontological structure, while $75 \%$ (3 out of 4 ) of the electron questions were leading. Further, $17 \%$ ( 2 out of 12 ) of the photon questions were coded as leading specifically towards blended or parallel ontologies, while it was $25 \%$ ( 1 out of 4 ) for the electron questions. Thus, these initial results suggest that the higher frequency of blended and parallel ontologies for photons is not due to a higher frequency of questions that we expect might guide students toward those structures. This is of course limited by small sample size (e.g., only four questions about electrons), and will be the subject of further investigation. Future work will explore not only how the content and wording of prompts plays a role in student reasoning, but also the forms of the questions and how they are framed in an instructional context.

We ask these questions specifically in a modern physics context, but it may be interesting to also explore how students' ontologies might be the same or different in an upper division QM course. In our analysis, we coded the responses from seven students who self-selected into our focus group study. While this small sample size is sufficient to demonstrate that we can identify different ontological structures in students' individual written work, the same patterns of use may not hold when the coding is expanded to the entire class. Such analyses will be presented in a future paper.

There are a few preliminary instructional implications we can derive from our results. When writing curricular materials or evaluating students' sense making, instructors ought to be aware that students can and do operate in multiple modes of reasoning about entities. Additionally, we suspect that the wording and framing of prompts may impact the ontological reasoning students engage in, which is important because the ontologies student use influence their learning.

\section{ACKNOWLEDGMENTS}

Thanks to the anonymous Modern Physics students for their participation in this research, and to the CU PER group for substantial feedback about data analysis. This work is supported by NSF grants No. 1322734, No. 1548924, No. 1625824, and a Graduate Research Fellowship.
[1] M. T. Chi and J. D. Slotta, Cognition and instruction 10, 249 (1993).

[2] J. D. Slotta and M. T. Chi, Cognition and instruction 24, 261 (2006).

[3] J. R. Hoehn and N. D. Finkelstein, Phys. Rev. Phys. Educ. Res. 14, 010122 (2018).

[4] A. Gupta, D. Hammer, and E. F. Redish, The Journal of the Learning Sciences 19, 285 (2010).

[5] B. W. Dreyfus, A. Gupta, and E. F. Redish, International Journal of Science Education 37, 812 (2015).
[6] J. R. Hoehn, N. Finkelstein, and A. Gupta, in Physics Education Research Conference 2016, PER Conference (Sacramento, CA, 2016) pp. 152-155.

[7] G. Fauconnier and M. Turner, The way we think: Conceptual blending and the mind's hidden complexities (Basic Books, 2008).

[8] S. McKagan, K. Perkins, and C. Wieman, in AIP Conference Proceedings, Vol. 883 (AIP, 2007) pp. 34-37.

[9] C. Baily and N. D. Finkelstein, Physical Review Special TopicsPhysics Education Research 11, 020124 (2015). 